\title{
LONG-TERM BRIGHTNESS CHANGES OF TWO CVS
}

\author{
D. A. SOKOLOV ${ }^{1}$, S. YU. SHUGAROV ${ }^{1}$, E. P. PAVLENKO ${ }^{2}$ \\ 1.Sternberg Astronomical Institute, Universitetsky avenue, 13 \\ 119899, MoscowV-234, Russia, e-mail: shugarov@sai.msu.su \\ 2.Crimean Astrophysical Observatory, Crimea 334413, Ukraine
}

\begin{abstract}
The long-term light curves of the AM Her type binary BY Cam and the nova-like variable PG $2133+115$ are presented. The $B V R$ observations were carried out at a $0.5 \mathrm{~m}$ telescope equipped with a high sensitive TV tube superisocon (Abramenko et al. 1978), the $U B V$ photoelectric data were obtained at 0.6 and $1.25 \mathrm{~m}$ telescopes and the photographic estimates were made from the negatives obtained at the $0.4 \mathrm{~m}$ astrograph in Crimea.

Both stars show high and low brightness states. They show similar behaviour in some respects: the amplitude between high and low states was about two magnitudes in $B$, but the duration of a low state was approximately two months for BY Cam and $1 \ldots 2$ years for PG $2133+115$.
\end{abstract}

\section{1. $P G 2133+115$}

Green, Ferguson \& Liebert (1982) noted that this star has a UV excess and so is possibly a CV. We obtained $U B V(1984-95)$ and photographic (196095) observations of this CV. On average, magnitudes and colours were as follows: $V=14.7 \mathrm{mag}, B-V=0.0 \mathrm{mag}, U-B=-0.9 \mathrm{mag}$. Misselt \& Shafter (1995) and the current authors did not detect the period of $2.9 \mathrm{~h}$, found earlier from radial velocity variations. According to the photographic observations in 1969-70 the object was 2 mag fainter than the mean level and $1 \mathrm{mag}$ light variations with a time-scale of $10 \ldots 20$ days were observed (see Fig. 1).

We suggest that PG $2133+115$ is a CV which is principally in the 'on' brightness state and is observed at a comparatively small inclination angle. 

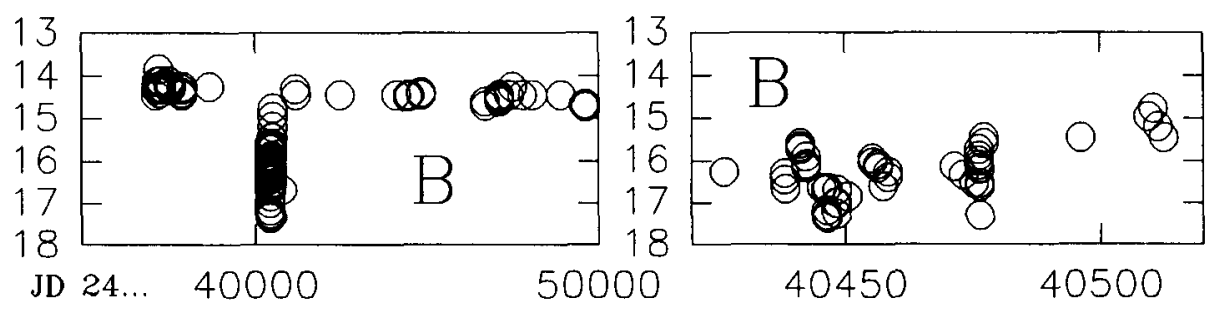

Figure 1. Left: PG $2133+115$ in 1960-94. Right: The low state in 1969.

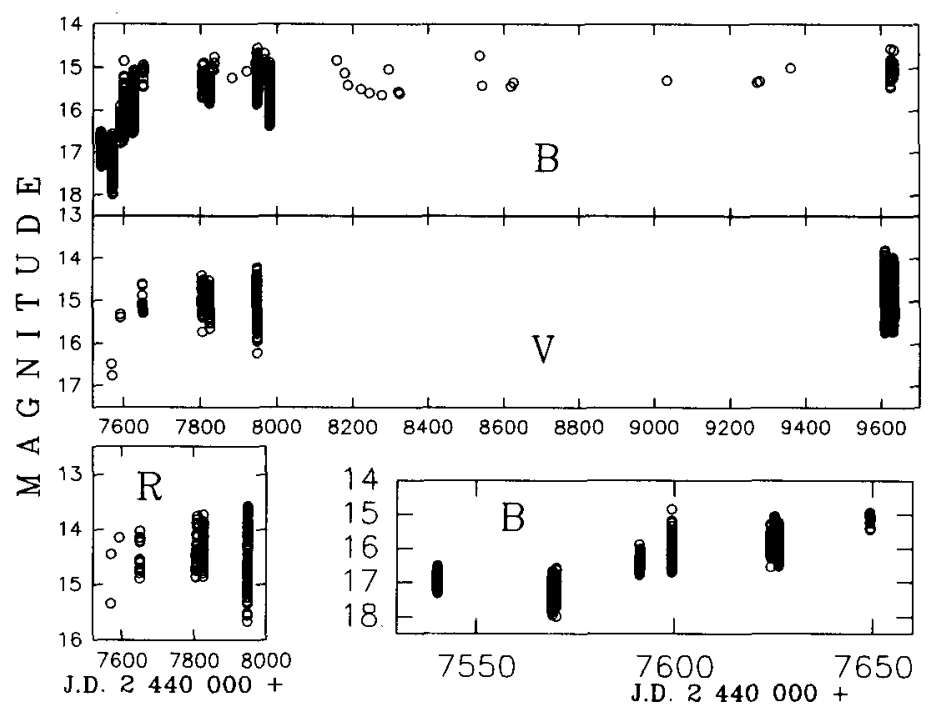

Figure 2. Left: BY Cam in 1989-94. Right: Low and intermediate states of BY Cam.

\section{BY Cam}

TV observations of BY Cam were obtained in 1989-90 and in 1994, photoelectric in 1994, and photographic estimates in 1953-93. The $B V R$ light curve is shown in Fig. 2 (left), which demonstrates the low and high brightness states. In more detail the low and intermediate states are shown in Fig. 2 (right). The low state duration was a brief episode lasting two months only, returning to the high brightness level during three months. The maximal amplitude of the long-term variations was detected in $B$ (2 mag).

\section{References}

Abramenko, A.M., Prokof'eva, V.V., Bondar, N.I. et al., 1988, Bull. of the Crimean Astroph. Obs., 78, 189

Green, R.F., Ferguson, D., Liebert J., 1982, PASP, 94, 560

Misselt, K.A., Shafter, A.W., 1995, AJ, 109, 1757 